\title{
Análise iconográfica do tópico evolução biológica em livros didáticos de Biologia para o ensino médio
}

\section{RESUMO}

Guilherme Kunde Braunstein zeneffi@gmail.com 0000-0002-1210-104X

Secretaria Estadual de Educação, Rio Grande do Sul, Brasil.

Marcelo Leandro Eichler exlerbr@gmail.com

0000-0001-5650-9218

Universidade Federal do Rio Grande do Sul, Porto Alegre, Rio Grande do Sul, Brasil.

\begin{abstract}
Os livros didáticos podem ser considerados um bom reflexo dos currículos escolares e possuem um importante papel como mediadores dos processos de ensino e de aprendizagem. Nesta pesquisa, a análise dos livros didáticos recaiu sobre os desenhos, os infográficos e os diagramas que são usados para apresentar o tema "evolução biológica", considerado o eixo central da biologia. A análise iconográfica desse assunto foi realizada em livros de biologia de ensino médio de autores com obras aprovadas pelo Programa Nacional do Livro Didático (PNLD 2012). A partir dessa análise, verificou-se que os livros apresentam poucas descrições e explicações sobre como diagramas e cladogramas devem ser lidos ou interpretados. Também se observou a preferência dos autores por diagramas que podem ser considerados de difícil compreensão. Apesar disso, pode-se notar que os autores têm apresentado diferentes modificações desses diagramas, melhorando-os, o que tem permitido uma melhor interpretação dos diagramas evolutivos.
\end{abstract}

PALAVRAS-CHAVE: Livros Didáticos. Análise Iconográfica. Evolução. Cladograma. 


\section{INTRODUÇÃO}

A literatura na área de ensino de ciências e de biologia considera o tema "evolução biológica" como essencial para se compreender a Biologia (ALMEIDA e FALCÃO, 2005; ENGELKE, 2009; MEYER e EL-HANI, 2005; SANDERS e NGXOLA, 2009; SANTOS e CALOR, 2007a e 2007b). Os parâmetros curriculares nacionais (PCNs), por sua vez, aconselham fortemente que os professores de Biologia no ensino médio utilizem o eixo ecologia-evolução como eixo integrador de todos os conteúdos dentro da Biologia (BRASIL, 2006, p. 22). Porém, existem diversos desafios que ainda precisam ser vencidos antes que se possa afirmar que durante as aulas de biologia a evolução está sendo usada como um eixo integrador.

Entre esses desafios é possível afirmar que a evolução apresenta ao aluno uma série de elementos e termos que podem ser considerados de difícil compreensão, estressantes e amedrontadores (SMITH, 2009). Esse tema costuma ser apresentado de modo fragmentado e fora de contexto, sendo restringindo geralmente a alguns poucos capítulos, apresentados no último ano da educação básica (ELLIS e WOLF, 2009; NEHM et al., 2008; SMITH, 2009). Em outros casos, o assunto evolução biológica nem ao menos é trabalhado em aula (MEIKLE, 2011; PRINOU, HALKIA e SKORDOULIS, 2008), ou é trabalhado focalizando temas menos controversos, como a microevolução, sendo essa uma das explicações para os modelos alternativos que os alunos elaboram para justificar a evolução de grandes grupos (BIZZO e EL-HANI, 2009).

Além disso, o ensino da evolução muitas vezes passa a ideia de um processo linear, em que uma espécie se transforma quase instantaneamente em outra, ignorando fatores temporais e históricos (BELLINI, 2006; ALMEIDA e FALCÃO, 2010), podendo entrar em conflito com as visões de mundo dos alunos (COSTA, MELO, TEIXEIRA, 2009). Sendo necessário que nesses momentos de conflito entre saberes científicos e da cosmovisão dos alunos, os professores consigam mediar o diálogo entre essas duas áreas (SCHILDERS et al., 2009).

Esse papel de mediador atribuído aos professores durante o ensino da evolução traz algumas barreiras adicionais. Entre elas, podem-se citar as carências da formação do professor, a falta de tempo para abordar o tema, a falta de materiais didáticos e a imaturidade dos alunos (ALMEIDA e FALCÃO, 2010; ZAMBERLAN e SILVA, 2009). Assim, seja por falta de acesso ou compreensão de conteúdos específicos durante a graduação, ou ainda pela falta de recursos didáticos, os professores acabam tendo que ensinar um assunto que não dominam totalmente (ABRIE, 2010; SANDERS e NGXOLA, 2009; TIDON e LEWONTIN, 2004), correndo o risco de incorrer em equívocos conceituais, ou simplesmente se omitirem de ensinar aspectos evolutivos que não compreendem ou com os quais não saibam lidar (FABRíCIO et al., 2006; SANDERS e NGXOLA, 2009; TIDON e LEWONTIN, 2004).

Dentro desse quadro, um dos recursos utilizados pelos os professores para auxiliá-los são os livros didáticos. Quando se fala em livros didáticos, é preciso considerar as referências apresentadas pelo Programa Nacional do Livro Didático (PNLD), programa do governo que seleciona livros didáticos a serem distribuídos nas escolas públicas do país. O PNLD para o ensino médio selecionou para o ano de 2012 uma série de 8 coleções de livros de Biologia, sugerindo como um dos temas articuladores da Biologia a origem e evolução dos seres vivos (BRASIL, 2011). 
Em relação ao ensino do tema evolução é proposto que seja dada ênfase aos aspectos chave da evolução, ao mesmo tempo em que se apliquem abordagens mais instigantes (NEHM e REILLY, 2007). Uma dessas abordagens é o uso de cladogramas como meio de apresentar de maneira lógica a origem, o desenvolvimento e a transformação de características fenotípicas e também genotípicas, permitindo também que se tenha uma visão mais ampla e não linear dos processos de especiação, por meio do qual se torna possível observar as irradiações e convergências em suas origens (SANTOS e CALOR, 2007a e 2007b).

\section{Análise iconográfica}

Dentro da biologia evolutiva algumas das representações utilizadas para sintetizar e facilitar a visualização das linhagens evolutivas são os cladogramas e as árvores filogenéticas. Os primeiros podem ser definidos como diagramas hierárquicos hipotéticos baseados em características em comum entre os táxons (CATLEY e NOVICK, 2008; NOVICK e CATLEY, 2007), enquanto que os últimos serian definidos, de modo semelhante, como sendo inferências às relações filogenéticas entre os organismos (BAUM e OFFNER, 2008).

O uso dos cladogramas no ensino é algo útil e prático por no mínimo três motivos: i) por simplificar o contexto ao descartar informações desnecessárias; ii) por tornar os conceitos abstratos mais concretos; e iii) por substituir modelos por outros recursos de mais fácil interpretação (NOVICK e CATLEY, 2007).

Essa capacidade dos diagramas de condensarem em si uma infinidade de características se torna evidente quando se verifica que com algumas poucas linhas se torna possível representar homologias, relações de ancestral-descendente, desenvolvimento de novidades evolutivas, ramificações de táxons e a ideia de tempo evolutivo (BAUM e OFFNER, 2008; CATLEY e NOVICK, 2008; FRANKLIN, 2010; MARCELOS e NAGEM, 2011; NOVICK e CATLEY, 2007).

Santos e Calor (2007a e 2007b) defendem de modo bem articulado o uso dos cladogramas como meio de integrar os diferentes conhecimentos biológicos, servindo de contexto para o ensino sobre os diferentes grupos biológicos, ao trazerem informações sobre a origem de estruturas e processos de diferenciação. A partir de então, indicam uma orientação para outros conteúdos a serem trabalhados em sala de aula pelos professores de Biologia. Esses autores propõem, por fim, que os cladogramas sejam construídos pelos próprios alunos.

A capacidade de visualizar a vida na terra e sua história contada em diagramas tem sido chamada de "pensamento de ramificação" (do inglês "tree thinkig") sendo apresentada como um requisito sem o qual seria impossível se compreender a evolução (BAUM e OFFNER, 2008; CATLEY e NOVICK, 2008; NOVICK e CATLEY, 2007; SANDVIK, 2008). Alguns autores sugerem que, para se evitar o desenvolvimento de visões equivocadas, o momento mais apropriado para o desenvolvimento desses pensamentos seria ao se começar a trabalhar com as características que os táxons têm em comum (MEIR et al., 2007; NOVICK e CATLEY, 2007).

Além disso, deve ser considerado, ao tratar desses diagramas, que a sua interpretação não ocorre de modo natural, uma vez que, na condição de um 
pensamentos evolutivos de ramificação presentes nos diagramas apresentam seus próprios desafios, precisando assim ser aprendidos pelos alunos, sendo o professor o orientador dessa nova leitura (METZGER, 2011; SANDVIK, 2008).

Dentro desse contexto de ensino, ademais das já apresentadas dificuldades relacionadas à formação dos professores, os próprios diagramas presentes nos livros didáticos poderiam ser uma fonte de confusão para os alunos, seja devido à simplificação de suas figuras, seja por darem margem para interpretações equivocadas (CATLEY e NOVICK, 2008; SANDVIK, 2008). Essas interpretações, por sua vez, podem levar ao desenvolvimento de ideias enganosas, como: i) a maior similaridade entre os grupos separados por menor número de nós do diagrama; ii) os organismos à esquerda das representações seriam os mais antigos; iii) a dificuldade para compreender que as linhagens descendem de nós em particular; iv) ou mesmo a utilização de um pensamento anagenético, segundo o qual os organismos se transformam em seus descendentes ao invés de darem origem a eles (CATLEY, NOVICK e SHADE, 2010; MEIR et al., 2007; NOVICK e CATLEY, 2007). Todas essas dificuldades se revelam e se agravam ainda mais quando se rotacionam os ramos dos diagramas, ou mesmo parte deles (BAUM e OFFNER, 2008; NOVICK e CATLEY, 2007).

Esses desafios de ensino acabam por merecer atenção ao se compreender a utilidade dessas representações. Uma vez que essas representações possibilitam que de modo rápido se tracem relações de parentesco evolutivo, permitindo que se expliquem processos de especiação e também que se estabeleçam relações entre os organismos atuais e os já extintos, assim como entre os organismos estudados em sua filogenia com grupos externos (FRANKLIN, 2010; MARCELOS; NAGEM, 2011), parece, portanto, oportuno analisar como essas representações aparecem nos livros didático de biologia para o ensino médio.

\section{Cladogramas nos livros didáticos de Biologia}

Por seu grande potencial explicativo, os cladogramas estão se tornando cada vez mais comuns nos livros-texto de Biologia. Neste sentido, o presente trabalho apresentará uma análise iconográfica dos diferentes tipos de cladogramas presentes em livros didáticos de Biologia destinados ao ensino médio. Nesta pesquisa, foram analisados livros de todos os autores aprovados pelo PNLD 2012 Biologia, sendo 5 deles volumes únicos, e 3 coleções em 3 volumes ${ }^{1}$ estando as obras apresentadas na Tabela 1, cada um desses livros foi lido na íntegra e teve suas figuras analisadas em termos de presença de termos evolutivos.

\footnotetext{
${ }^{1}$ Foi feita a opção de dar preferência a análise de obras em volume único de autores aprovados pelo PNLD ao invés das obras em si, as quais são sempre em 3 volumes, por a análise iconográfica aqui feitas ter sido realizada durante a dissertação de mestrado de Braunstein (2013), na qual as obras foram analisadas na integra também em seus textos. Desse modo com o objetivo de o autor dar conta da análise de todos os autores aprovados pelo PNLD, se deu preferência pela analise das obras em volume único, o que implicou em um significativo esforço extra que pode ser alocado nas análises das obras.
} 
volume único

Novas Bases da Biologia - Volumes Nélio Bizzo

Ática

1,2 e 3

Biologia: volume único

Biologia: volume único

Biologia: volume único

\section{César da Silva Júnior, Sezar Sasson e Nelson Saraiva Caldini Júnior}

Sérgio Linhares e Fernando Gewandsznajder Ática

Sônia Lopes e Sergio Rosso

Saraiva

Biologia: ensino médio, volume Antônio Carlos Pezzi, Demétrio Gowdak e FTD único Neide Mattos

Biologia: ensino médio - Volumes 1, Fernando Santiago dos Santos, João Batista SM

2 e3

Aguilar e Maria Martha de Oliveira

Biologia - Volumes 1, 2 e 3

J. Laurence e V. Mendonça

Nova Geração

Tabela 1 -Lista de livros analisado na pesquisa.

No que diz respeito à leitura e à classificação de cladogramas, Catley e Novick (2008) apresentam um excelente trabalho nesse sentido, o qual será seguido com algumas modificações para as análises iconográficas realizadas nesta pesquisa. As modificações em questão foram a exclusão das classes da tipologia para as quais não foram encontrados exemplos nos livros aqui analisados. Segundo os autores seria possível fazer, grosso modo, uma divisão dos cladogramas em dois tipos básicos: a) o cladograma em escada (Figura 1), que é formado por linhas diagonais que se ligam a outras linhas perpendiculares cujas extremidades representam os táxons e cujos nós representam a ramificação entre duas linhagens; e b) o cladograma em árvore (Figura 2), formado por linhas verticais que se ramificam em linhas horizontais ligadas em suas extremidades a outras duas linhas verticais ascendentes, sendo os táxons identificados pelas extremidades das linhas superiores e as ramificações identificadas pelas extremidade das linhas verticais abaixo daquelas que indicam os táxons. 


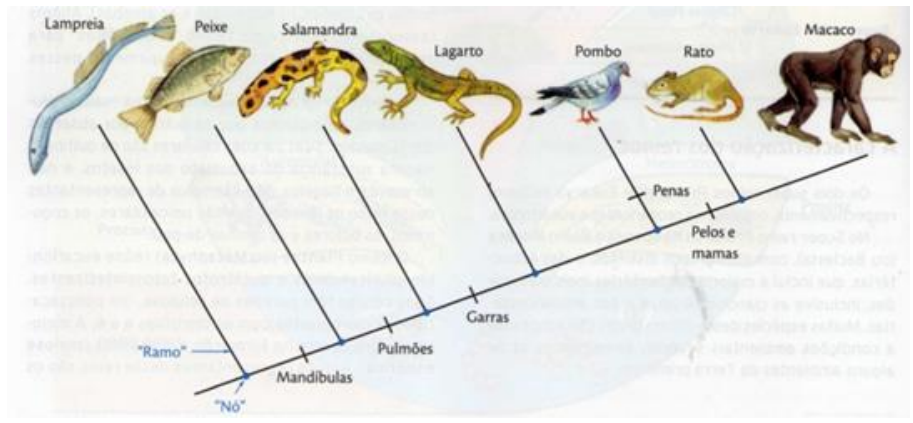

Figura 2 Cladograma em árvore com instruções (LINHARES e GEWANDSZNAJDER, 2007, p.

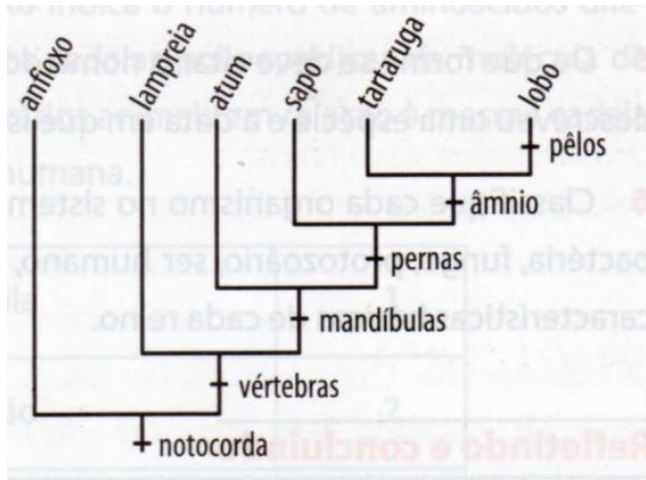

Esses dois modelos básicos poderiam dar origem a outras 15 variações, sendo os cinco primeiros tipos mutuamente excludentes: i) o cladograma semelhante ao em árvore, que diferiria do cladograma em árvore pela presença de alguma irregularidade (Figura 3); ii) o cladograma semelhante ao em escada, diferiria também pela presença de alguma irregularidade nas linhas (Figura 4); iii) o diagrama em árvore da vida seria uma representação de progressão de um grupo mais simples até o mais complexo (Figura 5); iv) o diagrama em anagênese, por sua vez, mostraria os descendentes lineares a partir de um único ramo basal (figura 6); por fim, v) os diagramas em outras formas de relação, seriam aqueles que não se encaixariam nos modelos anteriores, não havendo exemplos dele nos livros analisados. 
Figura 3 Cladograma semelhante ao em árvore (LOPES e ROSSO, 2005, p. 284)

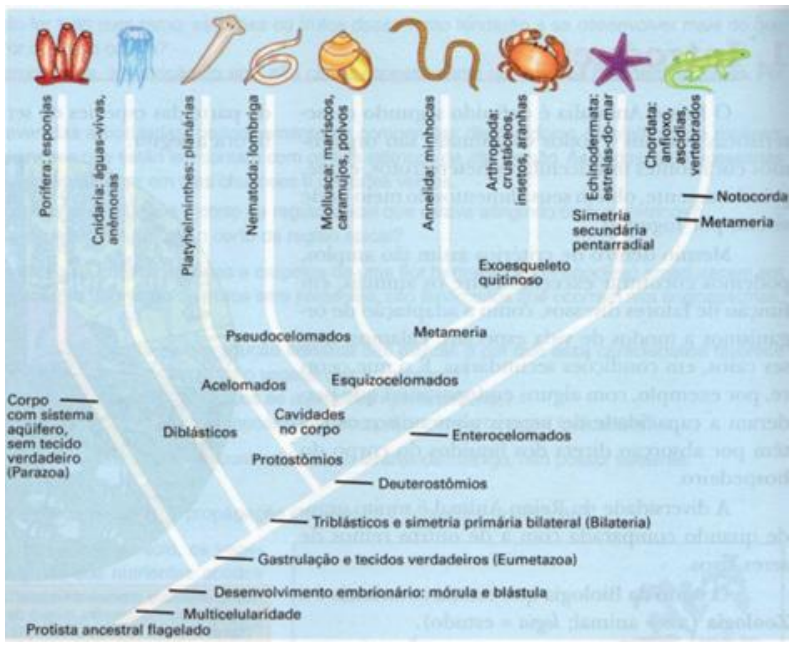

Figura 4 Cladograma semelhante ao em escada (LINHARES e GEWANDSZNAJDER, 2007, p. 219)

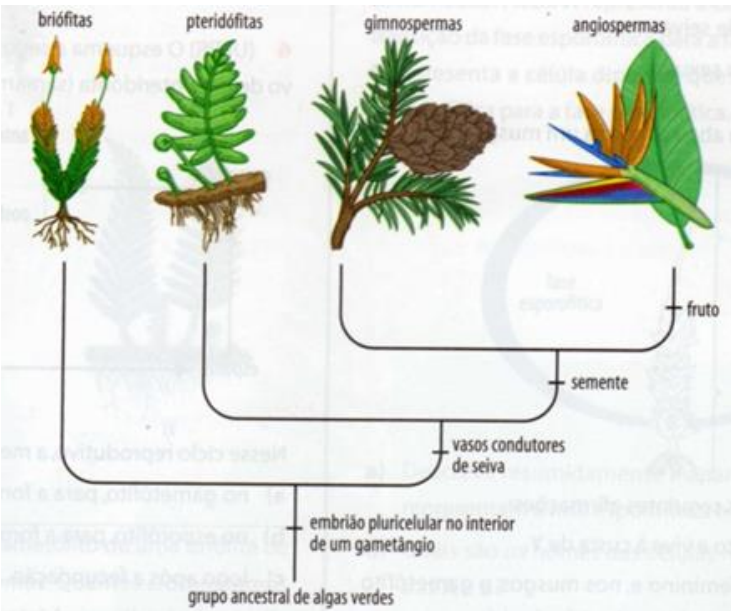


Figura 5 Diagrama em árvore da vida com ramificações laterais, transferência lateral e indicação de ancestral basal (LOPES e ROSSO, 2005, p. 189)

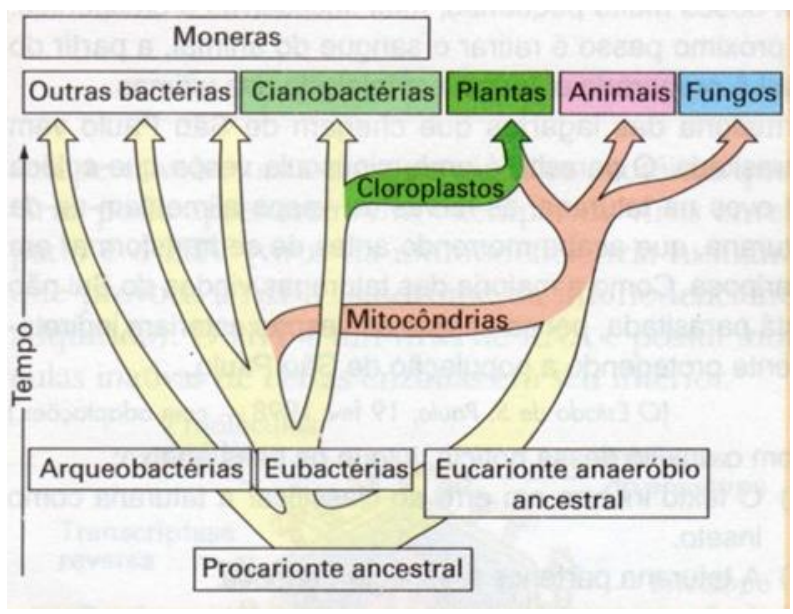

Figura 6 Diagrama em anagênese (SANTOS, AGUILAR e OLIVEIRA, 2010, p. 178)

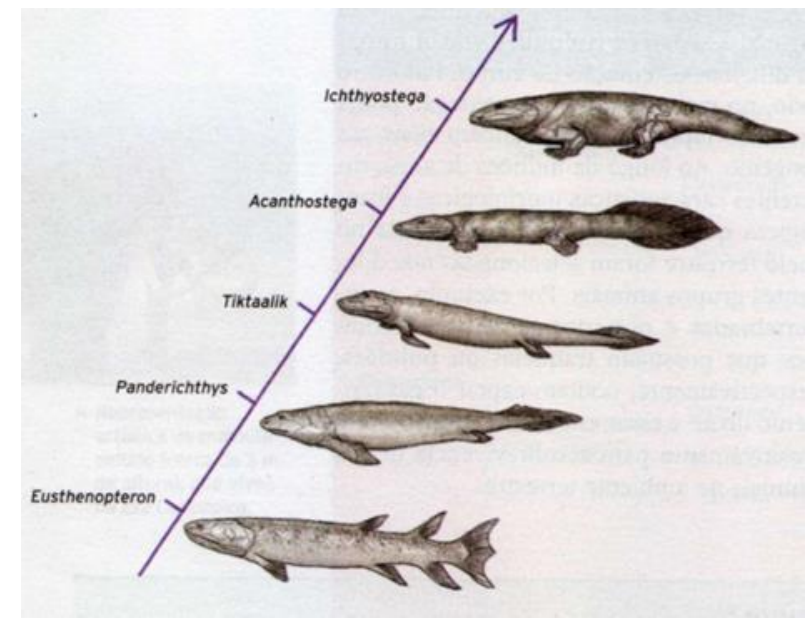

Os próximos três modelos a serem apresentados seriam variações dos últimos cinco: vi) o cladograma com ramificações laterais seria uma variação dos cinco tipos básicos que apresenta alguma politomia, ou seja, três ou mais linhagens saindo ao mesmo tempo de um mesmo ramo (Figura 5); vii) os cladogramas com ramos terminais acabando em diferentes pontos seriam cladogramas em escada ou árvore cujos ramos se encerrariam em alturas diferentes (Figura 7); viii) já os ramos não terminais marcados por táxons seriam ramos não terminais de cladogramas em escada, árvore ou árvore da vida que apresentariam nomes de táxons fora de suas extremidades terminais (Figura 7). 
Figura 7 Cladograma com ramos terminais acabando em diferentes pontos, marcados com táxons, com representações em barras da evolução dos hominídeos (AMABIS e MARTHO, 2006, p. 709)

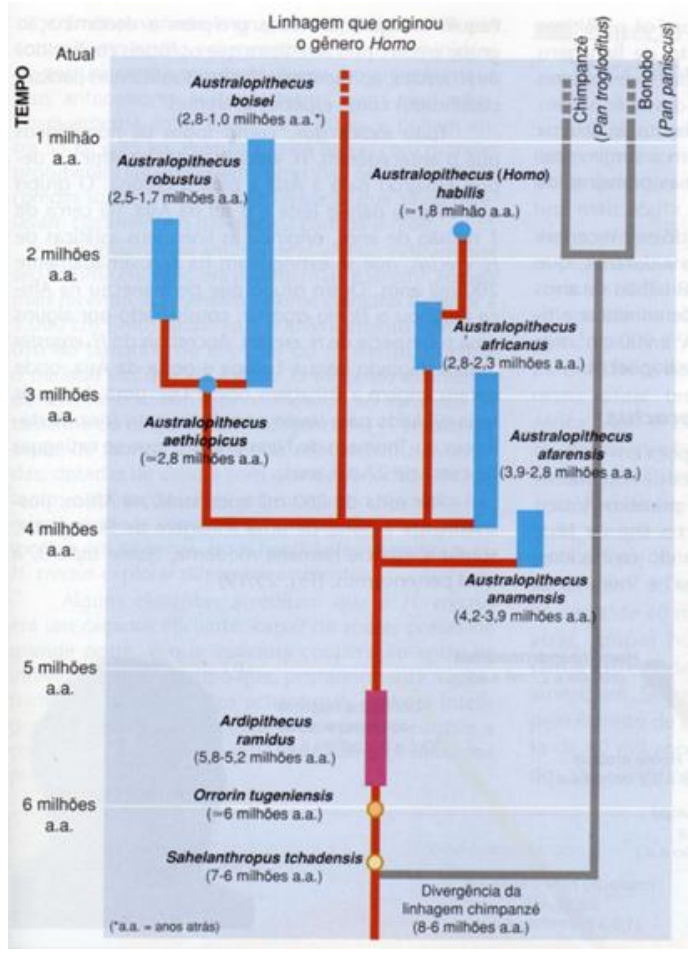

Figura 8 Cladograma com variação de espessura dos ramos e medida de tempo (BIZZO, 2010, p. 298)

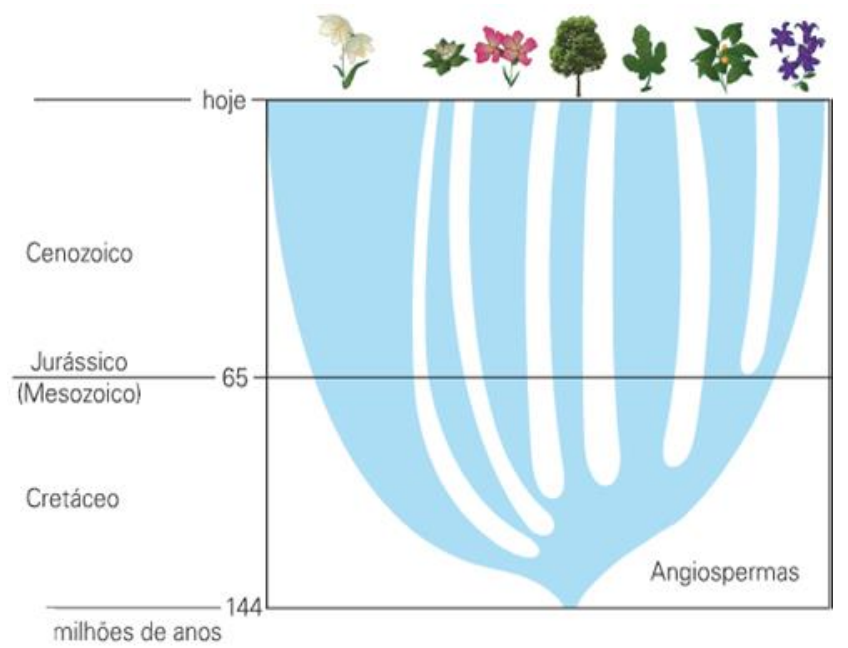


As últimas sete classificações seriam aplicáveis a qualquer dos modelos já apresentados, sendo em sua maioria auto-explicativas: 1) a transferência lateral seria uma indicação de hibridização ou passagem de material gênico entre linhagens (figura 5); 2) a representação em barras (figura 7); 3) a variação da espessura dos ramos (figura 8); 4) a rotulação de um nó basal com o nome de um ancestral (figura 5); 5) a indicação de tempo (figura 8); 6) o instrucional, que traz instruções da leitura do diagrama, tal como a indicação de novidades evolutivas (figura 2); e 7) a evolução dos hominídeos (figura 7).

Cada um desses critérios de análise foi aplicado a todas as figuras com conteúdo evolutivo ao longo da análise dos livros. Com base nos critérios de análise foi possível a formulação da Tabela 2, que mostra em sua última linha a proporção de figuras que mesmo falando da evolução não se encaixaram na definição de cladograma. As demais linhas da tabela apresentam a proporção de observações em cada classe que puderam ser encontradas em relação ao total de diagramas evolutivos analisados.

A análise dos dados tabelados revela que $20 \%$ das figuras consideradas evolutivas não são cladogramas. Quando são analisadas essas figuras, notou-se que as informações evolutivas se achavam nas legendas dessas, ou ao invés disso, apesar de a imagem passar uma ideia de evolução sua organização não permitiu que fossem vistas como diagramas do tipo cladograma. 
Tabela 2 - Porcentagem do item analisado referente ao total de diagramas analisados

\begin{tabular}{lccccccccc}
\hline & AM & PGM & LG & CSC & LR & ML & SAO & BI & Total \\
\hline Cladograma semelhante ao em árvore & $33 \%$ & 0 & $38 \%$ & 0 & $4 \%$ & 0 & 0 & $2 \%$ & $13 \%$ \\
Cladograma semelhante ao em escada & $56 \%$ & $40 \%$ & $54 \%$ & $27 \%$ & $57 \%$ & $61 \%$ & $37 \%$ & $37 \%$ & $45 \%$ \\
Árvore da vida & 0 & $20 \%$ & 0 & $9 \%$ & $13 \%$ & $15 \%$ & $26 \%$ & $9 \%$ & $12 \%$ \\
Anagênese & $11 \%$ & $20 \%$ & 0 & 0 & $17 \%$ & 0 & $16 \%$ & $3 \%$ & $8 \%$ \\
Outras formas de relação & 0 & $20 \%$ & $8 \%$ & $64 \%$ & $9 \%$ & $23 \%$ & $21 \%$ & $31 \%$ & $23 \%$ \\
\hline $\begin{array}{l}\text { Figuras que não foram classificadas } \\
\text { como diagramaa }\end{array}$ & 10 & 0 & 0 & 21 & 4 & 35 & 44 & 13 & 20 \\
\hline
\end{tabular}

Observação: abreviaturas dos nomes dos livros por nome de autores: Amabis e Martho (AM); Pezzi, Gowdak e

Mattos (PGM); Linhares e Gewandsznajder (LG); César, Sezar e Caldine (CSC); Lopes e Rosso (LR); Mendonça e

Laurence (ML); Santos, Aguilar e Oliveira (SAO); Nélio Bizzo (BI).

Uma primeira observação, não presente na tabela, é a de que não foi encontrado nenhum filograma nos livros analisados, ou seja, em nenhum dos diagramas o tamanho das dos ramos é proporcional ao tempo transcorrido entre cada ramificação. Isso poderia ser explicado pelo caráter introdutório dos livros, que aparentemente se preocupam mais em representar as ramificações evolutivas do que a questão de distância evolutiva entre os grupos. Entretanto, mesmo não havendo filogramas foi possível observar que $23 \%$ dos diagramas apresentaram alguma inferência temporal, o que é algo relevante já que as noções de tempo em evolução seriam uma importante barreira epistemológica a ser superada pelos alunos (BIZZO e BIZZO, 2006).

Dentre os diagramas, $45 \%$ deles se encaixaram melhor na classe de semelhantes a cladogramas em escada, sendo essa classe mais abundante do que a soma entre cladogramas semelhantes a árvore e diagramas em árvore da vida para cada um dos conjuntos de livros analisados. Tal informação é preocupante, já que apesar de as informações contidas nos cladogramas em escada e em árvore serem equivalente, a sua interpretação não o é, sendo os cladogramas em escada de mais difícil interpretação (CATLEY e NOVICK, 2008; NOVICK e CATLEY, 2007).

Mesmo que as publicações venham usando preferencialmente os cladogramas em forma de árvore, os textos destinados ao público leigo, tal como os livros didáticos, apresentam uma preferência pelo uso de cladogramas em escada, os quais seriam mais econômicos em termos de confecção (por serem desenhados com menos linhas do que os em árvore) e mais diretos no fornecimento de informações. Essa economia, porém, não deveria ser o suficiente para justificar a preponderância desse tipo de diagrama já que eles estariam envolvidos com um maior número de erros de interpretação, por suas linhas contínuas passarem uma ideia de eventos contínuos e ancestralidade comum a 
partir do grupo presente na linha principal, enquanto que o uso dos cladogramas em árvore poderia ser considerado mais intuitivo e também mais claro ao transmitir ideias de ancestralidade em comum (BAUM e OFFNER, 2008; MEIR et al., 2007; NOVICK e CATLEY, 2007). Voltando à Tabela 2, a desproporcionalidade entre o uso dessas duas representações fica evidente, percebendo-se que metade das obras não usa sequer um cladograma em árvore, além do que, em média há três vezes mais cladogramas em escada do que em árvore.

A preponderância dos cladogramas em escada poderia estar revelando ainda outro dado, por exemplo, algum tipo de distanciamento entre os autores e seu público alvo. Como já referido, os dois tipos de cladogramas são equivalentes quanto à quantidade de informações que os constituem, podendo ser igualmente bem interpretados por alguém familiarizado com essas representações, sendo assim, é provável que o predomínio dos cladogramas em escada nos livros didáticos reflita simplesmente a intenção dos autores de apresentar a representação aparentemente mais simples (o que seria verdade caso os alunos já conhecessem ou ao menos fossem previamente bem ensinados sobre o modo de se pensar em termos de ramificações evolutivas). Em suma, se por um lado a troca dos cladogramas em escada por cladogramas em árvore pode ser um meio de facilitar a aprendizagem dos alunos, por outro lado uma maior ênfase no aprendizado de se pensar em termos de ramificações evolutivas poderia igualmente auxiliar na solução desse problema.

Ao se comparar as limitações dos cladogramas com as de outros diagramas evolutivos, é perceptível uma carência de relações de ancestralidade em comum nos cladogramas, assim como uma maior ideia ligada a anagênese, progressividade, organização direcional, linear e teleológica nos demais diagramas (CATLEY, NOVICK e SHADE, 2010; CATLEY e NOVICK, 2008). De fato, ambas as apresentações seriam importantes para auxiliar a aprendizagem dos alunos, sendo assim, os resultados observáveis nos cinco grupos básicos da tabela revelam realidades diferentes entre os livros. Por exemplo, enquanto no livro CSC $73 \%$ dos diagramas (10 no total) não são cladogramas, apenas $8 \%$ dos diagramas apresentados em LG (1 único diagrama) não é apresentado na forma de cladograma. Mesmo que esses dados individualmente não possam indicar a superioridade em termos de compreensão da evolução biológica oferecida pelas obras, é possível afirmar que aquelas obras com mais diversidade e número de diagramas utilizados e também que apresentem maior proporção de cladogramas em árvore do que em escada sejam mais fortes candidatas a favorecer a compreensão dos seus leitores.

Outro ponto que deve ser levado em conta ao se avaliar os diagramas evolutivos diz respeito às variações feitas nos cinco tipos originais. Os ramos laterais presentes, por exemplo, em 33\% dos diagramas apresentados em AM, e em $60 \%$ dos diagramas analisados no livro PGM podem dificultar a compreensão dos alunos ao passarem uma ideia de ramificação não resolvidas (NOVICK e CATLEY, 2007). Sendo por isso importante que tais questões sejam bem compreendidas, a fim de que os diagramas não acabem por gerar mais confusão sobre o conteúdo trabalhado.

A noção de curso do tempo também vem à tona durante a leitura dos diagramas, fazendo com que a adição de escalas de tempo (absoluto ou relativo) auxilie os alunos a evitar equívocos, como o de considerar os grupos mais próximos 
fisicamente nas ilustrações também são os mais próximos em termos de história evolutiva (MEIR et al., 2007). Neste ponto é digno de nota que $23 \%$ dos diagramas trazem alguma escala de tempo, havendo livros como BI e AM que apresentaram escalas temporais em $37 \%$ e $33 \%$ dos diagramas, respectivamente.

Com referência à nomeação de táxons não terminais, a variação da espessura dos ramos, ao uso de barras, a nominação do grupo ancestral e à adição de instruções ou referências a novidades evolutivas, todas essas inserções podem alterar a capacidade de interpretação dos diagramas, facilitando-a quando bem empregadas (BAUM e OFFNER, 2008; MEIR et al., 2007). No caso dos livros analisados, $41 \%$ dos diagramas estavam acompanhados de alguma informação adicional à leitura, enquanto $19 \%$ nomearam o grupo basal. Destacando-se as obras ML, LR, e CSC por apresentarem respectivamente $62 \%, 61 \%$ e $55 \%$ dos diagramas com instruções adicionais de leitura.

Por fim, a evolução humana esteve presente em 11\% dos diagramas analisados, o que para metade dos autores correspondeu ao uso de uma única imagem, destacando-se, entretanto, o livro AM que dedicou a esse tema 3 dos 9 diagramas apresentados. A leitura desse resultado poderia indicar em um primeiro momento que o ser humano é tratado pelos autores como um entre tantos outros organismos resultantes da evolução. Porém, cabe ressaltar que todos os autores apresentam um capítulo ou parte de um capítulo dedicado à evolução humana, sendo assim, a ênfase dada para a evolução humana poderia ser mais bem avaliada por meio de uma análise descritiva das obras como um todo, sem se restringir às imagens apresentadas.

Como já referido, a leitura dos cladogramas e outros diagramas evolutivos depende em boa parte da capacidade dos alunos em pensar em termos de ramificação evolutiva, desse modo, outro aspecto levado em conta nas análises foi a presença e abrangência nos capítulos de textos destinados ao ensino da leitura de cladogramas e filogenias. Considerando-se o número de páginas destinadas a esse tema, bem como o número de parágrafos com essas explicações, formulouse a Tabela 3.

Tabela 3 - Número de parágrafos e páginas dedicados a explicação dos diagramas 


\begin{tabular}{lcccccccc} 
& AM & PGM & LG & CSC & LR & ML & SAO & BI \\
\hline Diagramas & 9 & 5 & 13 & 11 & 23 & 13 & 19 & 35 \\
Parágrafos & 10 & 3 & 2 & 5 & 40 & 24 & 6 & 5 \\
Páginas & 3 & 1 & 2 & 2 & 3 & 4 & 2 & 1
\end{tabular}

Observação: abreviaturas dos nomes dos livros por nome de autores: Amabis e Martho (AM); Pezzi, Gowdak e Mattos (PGM); Linhares e Gewandsznajder (LG); César, Sezar e Caldine (CSC); Lopes e Rosso (LR); Mendonça e Laurence (ML); Santos, Aguilar e Oliveira (SAO); e Bizzo (BI)

Tabela 3 - Número de parágrafos e páginas dedicados a explicação dos diagramas

A leitura da Tabela 3 revela que na maior parte dos casos as instruções sobre as interpretações dos diagramas é muito restrita, ocupando em média a 1,3 páginas por livro e 2,25 por autor (considerando os 5 livros em volume único e os 9 volumes das demais coleções), havendo apenas dois casos em que as obras dos autores ultrapassam a marca de 10 parágrafos de explicação. No que diz respeito ao uso de diagramas evolutivos, esses dados se mostram preocupantes, uma vez que mesmo que os autores tragam um grande número de diagramas em suas obras (35 no caso de $\mathrm{BI}$ ) haveria uma boa chance de o leitor não compreender totalmente as informações apresentadas pela falta de instruções para a leitura desse recurso, cuja interpretação não é natural, devendo, ao invés disso, ser aprendida pelos alunos individualmente (SANDVIK, 2008).

Ainda com base na tabela, merece atenção o livro $\mathrm{ML}$, que é o que mais páginas dedica ao tema (duas no volume dois e outras duas no volume três da coleção) e o segundo que mais traz parágrafos sobre a interpretação e construção de cladogramas. Por outro lado, o livro não se destaca pelo número de diagramas, aparentemente revelando a preocupação dos autores em apresentar uma melhor contextualização dos diagramas.

Além das análises já apresentadas nas duas primeiras tabelas, verificou-se também a quantidade de diagramas presentes dentro de cada unidade temática, localizando duas ocorrências nas partes de biologia geral, duas na parte de fisiologia, duas nas páginas destinadas às explicações sobre a origem da vida, 38 nos capítulos sobre Evolução e 86 nos capítulos sobre os grupos de seres vivos. Assim constata-se uma maior abundância desses termos nos capítulos sobre seres vivos do que nos destinados diretamente à evolução. Esse resultado pode ser explicado ao ponderarmos os dados por meio de uma comparação entre a porcentagem de diagramas em cada área e a porcentagem de páginas destinadas a cada tema, concluindo-se dessa forma que $66 \%$ dos diagramas estão localizados nos $32 \%$ das páginas que se dedicam aos seres vivos, enquanto que outros $29 \%$ dos diagramas estão localizados nos $7 \%$ dos livros que são dedicados à evolução. 
Dessa forma ao ponderarem-se os dados se conclui que os diagramas evolutivos estariam duas vezes mais concentrados nos capítulos sobre evolução do que nos sobre os seres vivos. Tais resultados estão dentro das expectativas, já que naturalmente se espera que se fale mais sobre evolução nos capítulos destinados a esse tema, bem como se espera que diagramas que tratem sobre a diversificação dos seres vivos (tal como cladogramas) sejam mais abundantes nos capítulos sobre esse tema do que em outros, como fisiologia, biologia geral ou mesmo as não citadas citologia e embriologia. Dessa forma pode-se concluir que a concentração maior de diagramas evolutivos está efetivamente nos capítulos sobre esse tema, sendo um ganho, porém, a grande presença desses também de modo diretamente aplicado aos seres vivos, o que dá uma utilidade prática para os conhecimentos apresentados, propiciando assim uma oportunidade maior para que os alunos integrem as diferentes áreas da Biologia por meio da evolução biológica.

Encerrando a análise iconográfica das imagens que fazem referência à evolução biológica, foi verificado que $80 \%$ dessas se apresentam na forma de diagramas. A prevalência desses modelos, por sua vez, sugeriu fortemente o uso de análises que levassem em conta as propriedades inerentes aos cladogramas e outros diagramas, as quais atestam para a necessidade de uma formação prévia voltada à capacitação da leitura dessas representações. Infelizmente a análise dos oito conjuntos de obras indica que tais instruções são uma grande carência para a maior parte dos livros didáticos analisados, situando a razão entre extensão de páginas dedicadas a essas explicações (PA) pelo número de páginas total (PT) entre as proporções de $0.006 \mathrm{PA} / \mathrm{PT}$ na obra LR e $0.001 \mathrm{PA} / \mathrm{PT}$ na obra $\mathrm{BI}$, ou seja, algumas obras usariam apenas pouco mais de um milésimo de seus textos explicativos para esse tema.

\section{Conclusões}

Inicialmente, sugeriu-se que o uso de diagramas pode ser considerado um bom instrumento para mediar o ensino da biologia através de um viés evolutivo. Por outro lado, para que tal instrumento de ensino seja realmente eficaz, faz-se necessário que os diagramas evolutivos sejam usados de modo claro e de fácil compreensão por parte dos educandos. A verificação dessa clareza pode, por exemplo, ser feita por meio da análise de livros didáticos destinados ao público específico do ensino médio, como foi o caso desta pesquisa.

Nesse sentido, foram analisados livros didáticos dos autores aprovados pelo Programa Nacional do Livro Didático de 2012. Verificou-se que, apesar das restrições apresentadas em termos de explicações sobre a interpretação dos diagramas, os cladogramas dessas obras estão em boa parte contextualizados por meio de referências a períodos de tempo, nome de grupos ancestrais e instruções de leitura, o que provavelmente compensaria em parte a carência de explicações interpretativas. Observou-se também o predomínio de representações de cladogramas em escada em detrimento dos cladogramas em árvore, o que pode ser considerado outra barreira para uma compreensão gráfica mais completa, que seria favorecida pelo primeiro tipo de cladograma.

Por fim, é perceptível a existência de uma variedade grande de diagramas alternativos aos cladogramas, fato positivo por oferecer uma maior variedade de opções explicativas aos leitores. A grande presença desses nos capítulos 
destinados a explicações sobre os seres vivos pode ser um ponto favorável ao uso prático da Biologia evolutiva como instrumento integrador dos conhecimentos. Por outro lado, apesar da variedade de modelos apresentados e das adaptações dos cladogramas favorecerem uma interpretação mais fidedigna do que se pretende apresentar, e de os diagramas estarem localizados dentro de suas próprias áreas de aplicação, o predomínio de cladogramas em escada e em especial a escassez de instruções sobre a interpretação dos diagramas podem ser barreiras para a aprendizagem dos alunos, que devem ser superadas com o auxílio e a mediação do professor na leitura e interpretação desse tipo de representação.

Obviamente uma análise iconográfica não é o suficiente para classificar em caráter final qual o melhor livro a ser adotado em uma escola, até por que essa escolha passa pela subjetividade do professor e por sua proximidade didática com os conteúdos presentes nos livros. Porém, verifica-se que diferentes critérios podem ser facilmente levados em conta pelo professor no momento em que esse fará a escolha da coleção a ser adotada. Alguns deles sendo a diversidade de diagramas, a prevalência de diagramas em árvore, além da constatação da maior abundância de diagramas nas unidades sobre seres vivos e evolução, fatores que podem agilizar uma leitura crítica baseada nesses critérios.

\section{Agradecimentos}

Ao CNPq, pela concessão da bolsa de pós-graduação do primeiro autor deste artigo, que permitiu a realização desta pesquisa. 


\title{
Iconographic analysis of biological evolution in biology high-school textbooks
}

\begin{abstract}
Textbooks can be considered a good reflection of school curricula and play an important role as mediators of teaching and learning processes. In this research, the textbook analysis involved the drawings, infographics and diagrams that are used to present the theme "biological evolution", considered the central axis of biology. Iconographic analysis was carried out in the high school biology textbooks of authors with works approved by the National Textbook Program. Being seen that the textbooks present few description and explanations about how the diagrams and cladograms should be read and interpreted. Also it observed the authors preference by diagrams that can be considered of hard understanding. Nevertheless, may be noted that this different modification in the diagrams, improving them, which has facilitate the interpretation of evolutionary diagrams.
\end{abstract}

KEYWORDS: Textbooks. Evolution. Cladogram. Iconographic Analysis. 


\section{REFERÊNCIAS}

ABRIE, A. Student teachers' attitudes towards and willingness to teach evolution in a changing South African environment. Journal of Biological Education, v. 44, n. 3, p. 102-107, 2010.

ALMEIDA, A.; FALCÃO, J. A estrutura histórico-conceitual dos programas de pesquisa de Darwin e Lamarck e sua transposição para o ambiente escolar. Ciência \& Educação, v. 11, n. 1, p. 17-32, 2005.

AMABIS, J.; MARTHO, G. Fundamentos da Biologia moderna: volume único. 4. ed. São Paulo: Editora Moderna, 2006.

BAUM, D.; OFFNER, S. Phylogenics \& Tree-Thinking. The American Biology Teacher, v. 70, n. 4, p. 222-229, 2008.

BELLINI, L. Avaliação do Conceito de Evolução nos Livros Didáticos. Estudos em Avaliação Educacional, v. 17, n. 33, p. 7-28, 2006.

BIZZO, N. Novas Bases da Biologia. São Paulo: Editora Ática, 2010.

BIZZO, N.; BIZZO, L. Charles Darwin in the Andes. Journal of Biological Education, v. 40, n. 2 , p. $68-73,2006$.

BIZZO, N.; EL-HANI, C. Darwin and Mendel : evolution and genetics. Journal of Biological Education, v. 43, n. 3, p. 108-114, 2009.

BRASIL. Orientações curriculares para o ensino médio: Ciências da natureza, matemática e suas tecnologias. Brasília: Ministério da Educação, Secretaria de Educação, v. 2, 2006.

BRASIL. Guia de livros didáticos : PNLD 2012 : Biologia. Brasília: Ministério da Educação. Secretaria de Educação Básica., 2011.

BRAUNSTEIN, G.K. A evolução biológica segundo os autores de livros didáticos de Biologia aprovados pelo Programa Nacional do Livro Didático (PNLD 2012): buscando um eixo integrador. 2013. 202f. Dissertação (mestrado em Educação em Ciências) Instituto de Ciências Básicas da Saúde: Universidade Federal do Rio Grande do Sul, Porto Alegre, 2013. 
CATLEY, K.; NOVICK, L.; SHADE, C. Interpreting evolutionary diagrams: When topology and process conflict. Journal of Research in Science Teaching, v. 47, n. 7, p. 861-882, 2010.

CATLEY, K.; NOVICK, L. Seeing the wood for the trees: An analysis of evolutionary diagrams in biology textbooks. BioScience, v. 58, n. 10, p. 976-987, 2008.

COSTA, L. D.; MELO, P. L.; TEIXEIRA, F. Evolução - tensões e desafios no Ensino Médio. Anais do VII ENPEC, p. 1-12, 2009.

DE ALMEIDA, A.; FALCÃO, J. As teorias de Lamarck e Darwin nos livros didáticos de Biologia no Brasil. Ciência \& Educação, v. 16, n. 3, p. 649-665, 2010.

ELLIS, M.; WOLF, P. Teaching "Species."Evolution: Education and Outreach, v. 3, n. 1, p. 89-98, 2009.

ENGELKE, D. Análise de livros didáticos de Biologia do Ensino Médio: estaria a teoria da evolução sendo um fio condutor? 2009. 27p. Trabalho de conclusão de curso - Licenciatura em ciências biológicas, Universidade Federal do Rio Grande do Sul, Porto Alegre, 2009.

FABRÍCIO, M. et al. A Compreensão das Leis de Mendel por Alunos de Biologia na Educação Básica e na Licenciatura. Ensaio Pesquisa em Educação em Ciências, v. 8, n. 1, p. 1-21, 2006.

FRANKLIN, W. Evolution \& Phylogenetic Analysis: Classroom Activities for Investigating Molecular \& Morphological Concepts. The American Biology Teacher, v. 72, n. 2, p. 114-121, 2010.

LINHARES, S.; GEWANDSZNAJDER, F. Biologia. 1a edição ed. São Paulo: Editora Ática, 2007.

LOPES, S.; ROSSO, S. Biologia: Volume único. 1. ed. São Paulo: Editora Saraiva, 2005.

MARCELOS, M.; NAGEM, R. Use of the "Tree" Analogy in Evolution Teaching by Biology Teachers. Science \& Education, v. 21, n. 4, p. 507-541, 2011.

MEIKLE, W. Banning Evolution. Evolution: Education and Outreach, v. 4, n. 3, p. 453-455, 26 ago. 2011. 
MEIR, E. et al. College Students' Misconceptions About Evolutionary Trees. The American Biology Teacher, p. 71-76, 2007.

METZGER, K. J. Helping Students Conceptualize Species Divergence Events Using the Online Tool "TimeTree: The Timescale of Life."The American Biology Teacher, v. 73, n. 2, p. 106-108, 2011.

MEYER, D.; EL-HANI, C. Evolução: o sentido da biologia. 1. ed. São Paulo: Editora UNESP, 2005.

NEHM, R. et al. Does the Segregation of Evolution in Biology Textbooks and Introductory Courses Reinforce Students' Faulty Mental Models of Biology and Evolution? Evolution: Education and Outreach, v. 2, n. 3, p. 527-532, 2008.

NEHM, R.; REILLY, L. Biology Majors' Knowledge and Misconceptions of Natural Selection. BioScience, v. 57, n. 3, p. 263, 2007.

NOVICK, L.; CATLEY, K. Understanding phylogenies in biology: the influence of a Gestalt Perceptual Principle. Journal of experimental psychology. Applied, v. 13, n. 4, p. 197-223, 2007.

PRINOU, L.; HALKIA, L.; SKORDOULIS, C. What Conceptions do Greek School Students Form about Biological Evolution? Evolution: Education and Outreach, v. 1, n. 3, p. 312-317, 2008.

SANDERS, M.; NGXOLA, N. Identifying teachers' concerns about teaching evolution. Journal of Biological Education, v. 43, n. 3, p. 121-129, 2009.

SANDVIK, H. Tree thinking cannot taken for granted: challenges for teaching phylogenetics. Theory in biosciences, v. 127, n. 1, p. 45-51, 2008.

SANTOS, C.; CALOR, A. Ensino de Biologia Evolutiva utilizando a estrutura conceitual da sistemática filogenética-II. Ciência \& Ensino, v. 2, n. 1, 2007a.

SANTOS, C.; CALOR, A. Ensino de Biologia Evolutiva utilizando a estrutura conceitual da sistemática filogenética-I. Ciência \& Ensino, v. 2, n. 1, 2007b.

SANTOS, F.; AGUILAR, J.; OLIVEIRA, M. Ser Protagonista Biologia. São Paulo: Edições SM, 2010. 
SCHILDERS, M. et al. Worldviews and evolution in the biology classroom. Journal of Biological Education, v. 43, n. 3, p. 115-120, 2009.

SILVA JÚNIOR, C. DA; SASSON, S.; CALDINI JÚNIOR, N. Biologia: volume único. 5. ed. São Paulo: Editora Saraiva, 2011.

SMITH, M. Current Status of Research in Teaching and Learning Evolution: II. Pedagogical Issues. Science \& Education, v. 19, n. 6-8, p. 539-571,2009.

TIDON, R.; LEWONTIN, R. Teaching evolutionary biology. Genetics and Molecular Biology, v. 27, n. 1, p. 124-131, 2004.

ZAMBERLAN, E.; SILVA, M. O evolucionismo como princípio organizador da Biologia. Temas \& Matizes, n. 15, p. 27-41, 2009.

Recebido: 2015-05-08

Aprovado: 2016-12-01

DOI: 10.3895/rbect.v10n2.3005

Como citar: BRAUNSTEIN, G. K.; EICHLER, M. L. Análise iconográfica do tópico evolução biológica em

livros didáticos de Biologia para o ensino médio. Revista Brasileira de Ensino de Ciência e Tecnologia, v.

10, n. 2, 2017. Disponível em: <https://revistas.utfpr.edu.br/rbect/article/view/3005>. Acesso em: xxx. Correspondência:

Direito autoral: Este artigo está licenciado sob os termos da Licença Creative Commons-Atribuição 4.0 Internacional. 